\title{
Clinical and Genetic Profiles in Chinese Patients with Huntington's Disease: A Ten-year Multicenter Study in China
}

\author{
Hong-Lei Li ${ }^{1, \#, ~ X i a o-Y a n ~ L i ", ", ~ Y i ~ D o n g, " \#, ~ Y a n-B i n ~ Z h a n g ², ~ H o n g-R o n g ~ C h e n g ', ~ S h i-R u i ~ G a n ², ~}$ \\ Zhi-Jun Liu ${ }^{3}$, Wang Ni', Jean-Marc Burgunder ${ }^{4, \&}$, X. William Yang ${ }^{5}$, Zhi-Ying Wu ${ }^{1,6, *}$
}

\begin{abstract}
${ }^{1}$ Department of Neurology and Research Center of Neurology in Second Affiliated Hospital, and Key Laboratory of Medical Neurobiology of Zhejiang Province, Zhejiang University School of Medicine, Hangzhou, China. ${ }^{2}$ Department of Neurology, First Affiliated Hospital, Fujian Medical University, Fuzhou, China. ${ }^{3}$ Department of Neurology, Huashan Hospital, Shanghai Medical College, Fudan University, Shanghai, China. ${ }^{4}$ Swiss Huntington's Disease Centre, Siloah, Gümligen and, Department of Neurology, University of Bern, Bern, Switzerland. ${ }^{5}$ Center for Neurobehavioral Genetics, Jane and Terry Semel Institute for Neuroscience and Human Behavior, Department of Psychiatry and Biobehavioral Sciences, Brain Research Institute, David Geffen School of Medicine, University of California at Los Angeles, CA, USA. ${ }^{6}$ Joint Institute for Genetics and Genome Medicine between Zhejiang University and University of Toronto, Zhejiang University, Hangzhou, China
\end{abstract}

[Received August 1, 2018; Revised September 9, 2018; Accepted September 11, 2018]

\begin{abstract}
Huntington's disease (HD) is an autosomal dominant inherited neurodegenerative disorder caused by CAG triplet repeats expansion in exon 1 of the Huntingtin gene (HTT). In China, HD is considered to have a low prevalence. The goal of this study was to describe the clinical characteristic and genetic profiles of HD in a Chinese cohort. A total of 322 individuals with expanded CAG repeats were consecutively recruited from the neurologic clinics of three medical centers in Southeastern China between 2008 and 2018. Among them, 80 were pre-symptomatic mutation carriers and 242 were symptomatic patients. The mean age at onset (AAO), defined here as the age at motor symptom onset, of the 242 manifest HD individuals was $40.3 \pm 11.9$ years and the mean CAG repeat length was $46.1 \pm 7.5$ in the group of symptomatic patients. Initial symptoms were abnormal movements in $88.8 \%$ of the patients with psychiatric symptoms in $6.2 \%$, cognitive impairment in $3.3 \%$ and others in $1.7 \%$. The AAO of motor was negatively correlated with the CAG repeat length in an exponential regression analysis $(R 2=0.74, P<0.001)$. Analysis of 46 parent-child pairs showed that the $C A G$ repeat length was longer in the offspring group $(45.8 \pm 7.6)$ than in the parent group $(43.8 \pm 3.0)(p=0.005)$. Overall, this study provides clinical and genetic profiles in a cohort of Chinese patients with $\mathrm{HD}$, which should contribute to a better understanding of this disorder.
\end{abstract}

Key words: Huntington's disease, Chinese population, phenotype, genotype

Huntington's disease (HD) is an autosomal dominant inherited neurodegenerative disorder characterized by movement disorder (i.e. chorea and dystonia), cognitive decline and psychiatric symptoms. HD is caused by CAG triplet repeats expansion in the exon 1 of the Huntingtin gene (HTT) [1]. Symptoms usually begin insidiously at mid-adult life, and inexorably worsen until patients' premature death, usually in about 15-20 years after the

*Correspondence should be addressed to: Dr. Zhi-Ying Wu, Department of Neurology and Research Center of Neurology in Second Affiliated Hospital, Zhejiang University School of Medicine, Hangzhou 310009, China. E-mail: zhiyingwu@ zju.edu.cn. \#Hong-Lei Li, Xiao-Yan Li and Yi Dong contributed equally to this work. ${ }^{\&}$ Jean-Marc Burgunder is currently a visiting professor at Sichuan University (Chengdu), Central South University (Changsha) and Sun Yat Sen University (Guangzhou) in China.

Copyright: @ $2018 \mathrm{Li} \mathrm{HL}$ et al. This is an open-access article distributed under the terms of the Creative Commons Attribution License, which permits unrestricted use, distribution, and reproduction in any medium, provided the original author and source are credited. 
motor symptom onset [2]. In general population, the normal HTT gene contains a sequence of 6-26 CAG repeats. Individuals who inherit an expanded CAG repeat of 40 or more will manifest the disease with a complete penetrance, whereas those who inherited 36-39 CAG repeats may be affected with milder penetrance. Although the intermediate alleles (27-35 CAG repeats) are considered not to be in a pathogenic range, they are unstable alleles and may expand or contract during the germline transmission [3].

HD prevalence is about $4-10$ per 100,000 in the Caucasian populations but varies greatly between ethnic origins [4]. Among East Asians and South Africans, the prevalence of $\mathrm{HD}$ is reportedly to be only $0.1-1$ per 100,000 [5-7]. Recently, an epidemiological study found the average annual incidence rate of HD in Taiwan China to be $0.1 / 100,000$ [8]. In the absence of a nationwide epidemiological study in mainland China, the prevalence of HD in the Han Chinese population remains unknown. Further, there is a paucity of clinical phenotype description in this population.

In this study, we aim to provide a description of the clinical characteristics, genetic profile in a cohort of Han Chinese HD patients and gene carriers. We compared our findings with those reported in other ethnic populations around the world. These data are of great significance in guiding the clinical practice, especially in practical diagnostic process and genetic counselling. Moreover, the clinical and genetic studies of HD in different ethnic populations may provide new insights into this disorder.

\section{MATERIALS AND METHODS}

\section{Subjects}

A total of 322 individuals with expanded triplet repeats (80 pre-symptomatic mutation carriers and 242 manifest HD) from 238 families were consecutively recruited from the neurologic clinics of three medical centers (Second Affiliated Hospital of Zhejiang University School of Medicine, First Affiliated Hospital of Fujian Medical University and Huashan Hospital affiliated to Fudan University) in China between February 2008 and March 2018. HD diagnosis was made by at least two senior neurologists according to patients' clinical manifestations in the context of positive HTT genetic tests. Predictive tests were performed in relatives as they requested after appropriate genetic counsel. Pre-symptomatic mutation carriers refer to those who show no motor signs and no other subjective symptoms or clinical sign which would be typical for HD. Comprehensive demographic and clinical data, including medical and psychiatric history, HD family history, and current medications were collected from the participants. All participants signed the informed consent form, or if the participants were unable to provide consent, an authorized representative provided the consents on their behalf. The study was approved by local Ethics Committees (i.e. Institutional Review Board) for medical research at three participating hospitals.

The age at onset (AAO) is defined by the age when motor signs or symptoms occur. Homozygous HD gene carriers refers to individuals with two expanded alleles.

\section{Genetic analysis of the HTT gene}

Genomic DNA was extracted from peripheral blood treated by ethylene diamine tetraacetic acid (EDTA). Genetic testing for HTT gene was performed using the method previously reported [9]. Briefly, the primers of $H T T$ (F: 5' -CAGAGCCCCATT CATTGCC-3' and R: 5'TGAGGAAGCTGAGGA GGC-3') were used to amplify the exon 1 in the CAG polymorphic region of the HTT gene. Polymerase chain reaction (PCR) amplicons were electrophoresed on a $1.5 \%$ agarose gel at $180 \mathrm{~V}$ for twenty minutes to determine the primary HTT results. Then the length of CAG repeats was determined by Sanger sequencing.

\section{Statistical analyses}

Demographic, clinical characteristics and genetic profile were analyzed using descriptive statistics. The data distributions of AAO and CAG were assessed by Kolmogorov-Smirnov test. AAO was normally distributed yet the CAG was distributed with a skew. For comparison between two continuous variables, $\mathrm{T}$ tests, Wilcoxon tests or Mann-Whitney were used as appropriate. ANOVA was used for comparison between multi-group. Correlation was calculated by Pearson or Fisher exact Chi-Square when appropriate. Statistical analysis was conducted using SPSS 20.0. The p-value less than 0.05 was considered statistically significant.

Table 1. The profile of persons with HD in our cohort.

\begin{tabular}{cc}
\hline & Number \\
\hline Patients & 322 \\
Manifest HD & 242 \\
Premanifest HD & 80 \\
Family & 238 \\
Clear transmit mode & 178 \\
Paternal/ Maternal & $95 / 83$ \\
Uncertain family history & 34 \\
Unknown family history & 26 \\
CAG available & 309 \\
\hline
\end{tabular}




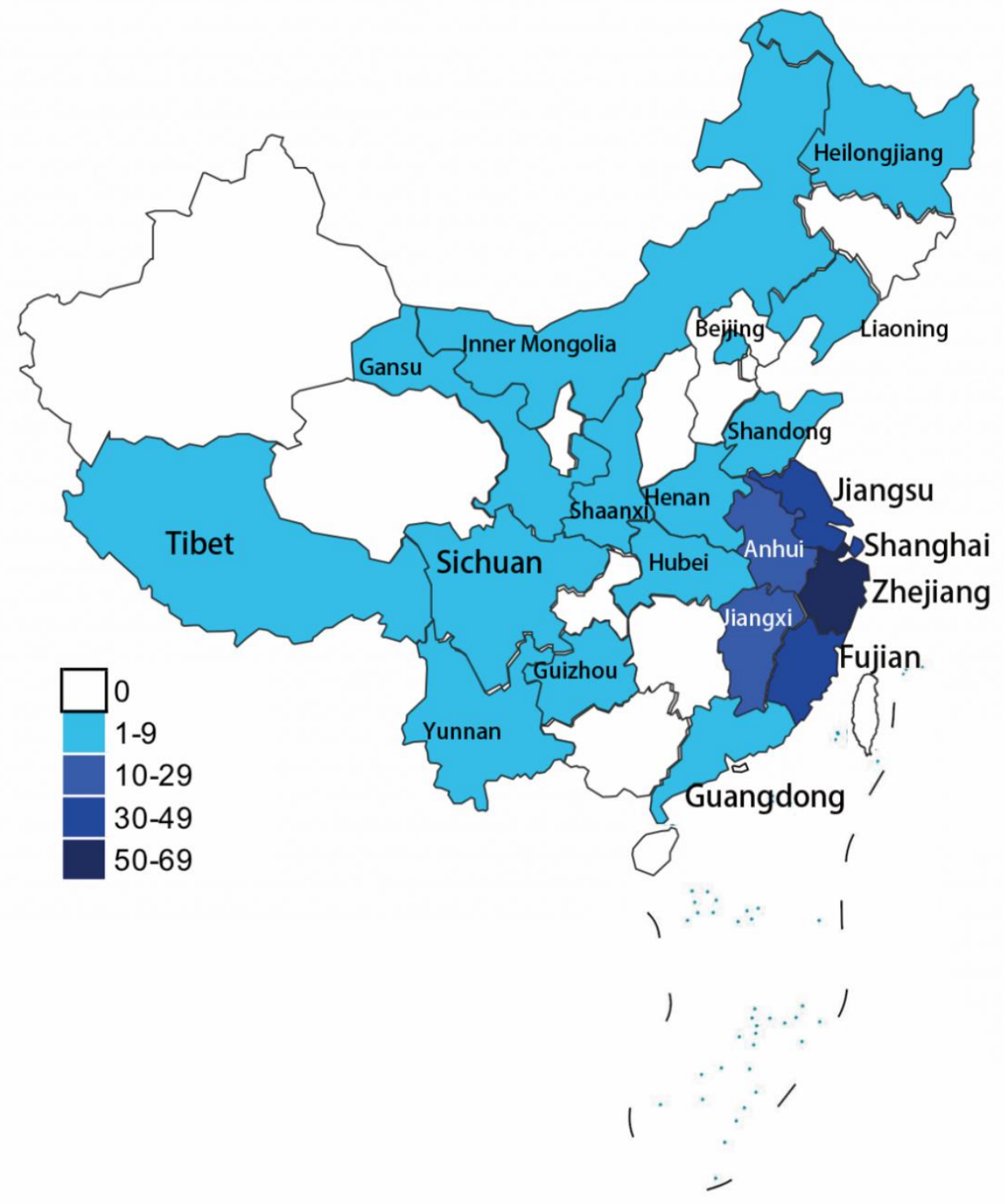

Figure 1. The geographical distribution of HD patients. Most patients were from Southeast China.

\section{RESULTS}

\section{Population features}

As shown in Table 1, among the 322 persons with abnormal repeats from 238 families, 242 (131 males and 111 females) were symptomatic patients while 80 (35 males and 45 females) were pre-symptomatic mutation carriers. To control against bias created by the presence of several family members, the index case from each family was included to calculate the transmission mode. Among 238 families, 178 had clear transmission mode $(95$ paternal and 83 maternal inheritance) and 34 denied their parents had any known chorea-like movements and information from the remaining 26 was lacking. Data on CAG repeat numbers was obtained from 309 subjects. DNA was missing from the remaining 13 persons. The mean age at genetic test was $29.9 \pm 11.7$ years (range 166 years) in the pre-symptomatic carriers with their mean number of CAG repeats of $44.2 \pm 4.0$ (range 37-59). The majority of the families were from Southeastern China (Fig. 1). The Unified Huntington's Disease Rating Scale (UHDRS) was applied to assess the motor, cognitive and psychiatric symptoms. Age at onset was assessed in a structured interview and by use of all clinical reports. Assessments were performed by trained neurologists. 
Table 2. Age at motor onset and CAG repeats among juvenile HD, adult HD and elder HD.

\begin{tabular}{|c|c|c|c|c|c|c|c|c|}
\hline & \multicolumn{2}{|c|}{ AAO (years) } & \multicolumn{2}{|l|}{ CAG } & \multicolumn{2}{|c|}{$\begin{array}{c}\text { Age at diagnosis } \\
\text { (years) }\end{array}$} & \multicolumn{2}{|c|}{ DOD (years) } \\
\hline & Mean (SD) & Range & Mean (SD) & Range & Mean (SD) & Range & Mean (SD) & Range \\
\hline Total $(n=242)$ & $40.3(11.9)$ & $4-71$ & $46.1(7.5)$ & $38-104$ & $45.7(12.4)$ & $7-83$ & $5.4(4.3)$ & $0.25-30$ \\
\hline male & $39.7(12.8)$ & $8-71$ & $46.5(8.0)$ & $39-104$ & $45.1(13.4)$ & $10-83$ & $5.5(4.4)$ & $0.25-27$ \\
\hline female & $41.0(11.0)$ & $4-60$ & $45.6(6.9)$ & $38-92$ & $46.4(11.2)$ & $7-68$ & $5.4(4.2)$ & $0.25-30$ \\
\hline Juvenile HD $(n=15)$ & $14.5(4.8)$ & $4-20$ & $67.0(16.8)$ & $46-104$ & $20.9(9.3)$ & $7-42$ & $6.4(6.9)$ & $1-27$ \\
\hline male & $14.8(4.2)$ & $8-20$ & $65.8(18.6)$ & $46-104$ & $21.2(10.2)$ & $10-42$ & $6.4(8.3)$ & $1-27$ \\
\hline female & $14.0(6.2)$ & $4-19$ & $69.0(14.8)$ & $56-92$ & $20.4(8.3)$ & $7-28$ & $6.4(3.3)$ & $3-10$ \\
\hline Adult HD $(n=220)$ & $41.4(9.4)$ & $21-59$ & $44.9(3.6)$ & $39-58$ & $46.7(10.1)$ & $23-68$ & $5.3(4.1)$ & $0.25-30$ \\
\hline male & $40.7(9.8)$ & $21-59$ & $45.2(3.5)$ & $39-58$ & $46.1(10.6)$ & $23-68$ & $5.4(3.9)$ & $0.25-23$ \\
\hline female & $42.0(9.1)$ & $21-59$ & $44.6(3.4)$ & $40-54$ & $47.3(9.5)$ & $26-68$ & $5.3(4.3)$ & $0.25-30$ \\
\hline Elderly-onset HD (n=7) & $63.7(4.8)$ & $60-71$ & $39.3(0.9)$ & $38-41$ & $69.6(6.7)$ & $63-83$ & $5.8(3.2)$ & $3-12$ \\
\hline male & $63.7(4.9)$ & $60-71$ & $39.3(0.9)$ & $38-41$ & $70.8(7.7)$ & $63-83$ & $5.6(3.8)$ & $3-12$ \\
\hline female & 60 & 60 & 38.5 & $38-39$ & 66.5 & $65-68$ & 6.5 & $5-8$ \\
\hline
\end{tabular}

DOD: Duration from onset to diagnosis

\section{Clinical spectrum}

\section{$\underline{\text { Age at onset }}$}

Reliable data on AAO were available in $242 \mathrm{HD}$ patients. The mean motor AAO was $40.3 \pm 11.9$ (range $4-71$ ) years (Table 2) (Fig. 2A). The average age at diagnosis was 45.7 \pm 12.4 (range $7-83$ ) years with an average diagnostic delay of 5.4 years. Fifteen $(6.2 \%)$ had a juvenile onset with a mean AAO of $14.5 \pm 4.8$ (range 7-20) years, and 7 (2.9\%) had an old age onset with a mean AAO of $63.7 \pm 4.8$ (range 60-71) years. The other 220 patients $(90.9 \%)$ had adult onset with an average mean AAO of $41.4 \pm 9.4$ (range 21-59) years. (Table 2).

\section{Spectrum of initial symptoms}

Among the 242 symptomatic gene carriers, 215 first presented with motor signs $(88.8 \%, 117$ males and 98 females) and 27 (11.2\%, 14 males and 13 females) presented with non-motor symptoms and developed motor disorder later. Chorea was the most frequent motor sign $(n=195,80.6 \%)$. Other types of motor symptoms included ataxia $(n=7,2.9 \%)$, gait instability $(n=9,3.7 \%)$, parkinsonism $(\mathrm{n}=1,0.4 \%)$, tics $(\mathrm{n}=1,0.4 \%)$, tremors $(\mathrm{n}=1$, $0.4 \%)$ and dystonia $(n=1,0.4 \%)$. Eight patients $(3.3 \%, 7$ males and 1 females) had cognitive impairment first, of whom 5 with memory decline and 3 with intellectual decline. Fifteen subjects $(6.2 \%, 4$ males and 11 females) had psychiatric and behavioral symptoms, including agitation $(n=9,3.7 \%)$, depression $(n=5,2.1 \%)$ and psychosis $(n=1,0.4 \%)$. In addition, two patients had mixed psychiatric and cognitive impairment at onset and one patient had concurrent movement disorder and cognitive decline. Finally, one juvenile patient presented with seizure as initial symptom (Fig. 2B). Gender had a significant different distribution in motor symptoms group vs psychiatric symptom group $(\mathrm{p}=0.04)$, or cognitive symptom group vs psychiatric symptom group $(\mathrm{p}=0.01)$ as females had a higher frequency in psychiatric symptom group compared with the other groups. However, there was no difference for gender between motor and cognitive groups $(\mathrm{p}=0.08)$.

\section{Duration of disease}

Data of 97 deceased HD patients from 238 families were analyzed to estimate the duration of disease. Seven of them died by accident $(n=2)$, suicide $(n=2)$, cerebral hemorrhage $(n=2)$ or because of esophageal cancer $(n=1$, at the age of 62). Hence, these seven patients were not included in the further analysis of disease duration.

As shown in Table S1, the mean duration of disease was $13.3 \pm 6.3$ (range 6-53) years defined by the mean AAO (42.9 \pm 10.4 years, range $21-70$ years) and mean age at death (56.2 \pm 11.1 years, range $38-83$ years). The 
median duration of disease was 11 years. To examine whether duration of disease was related to AAO, we divided participants into three groups: 20-39 years, $40-59$ years and 60 years and older. There was no difference between these three groups $(\mathrm{p}=0.50)$ with a mean disease duration of $14.1 \pm 8.6,13.3 \pm 5.0$ and $11.6 \pm 2.1$ years, respectively. There was no effect of gender on duration of survival $(\mathrm{p}=0.71)$.
A

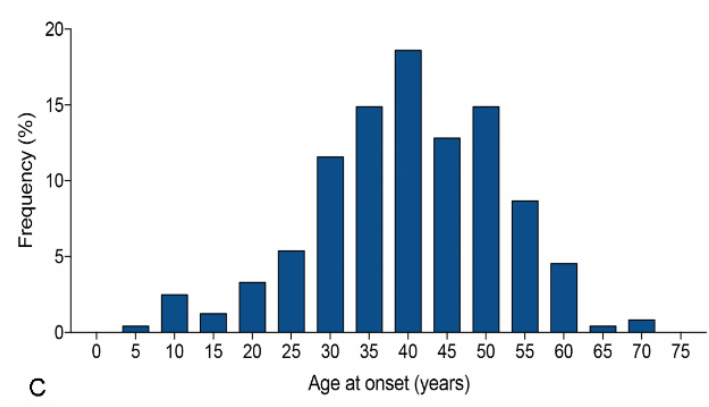

C

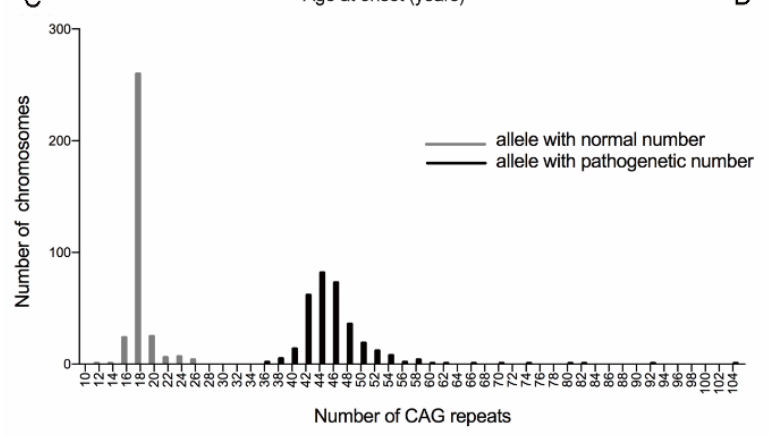

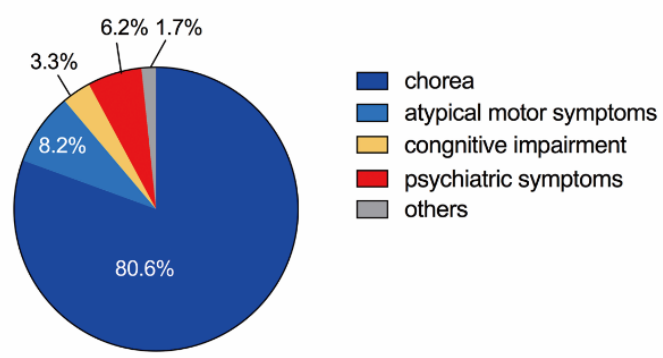

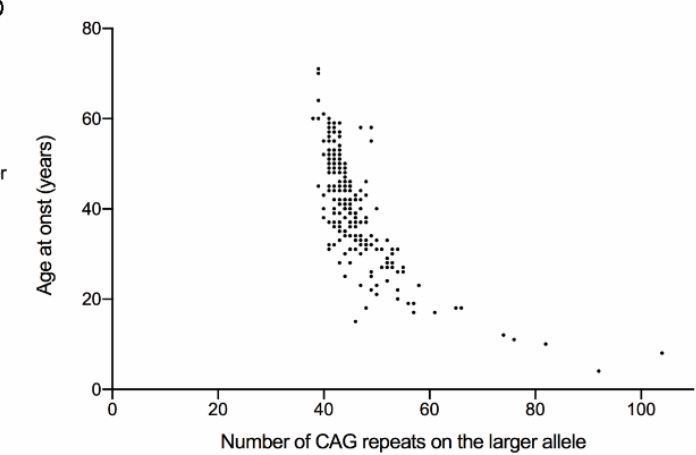

Figure 2. Clinical and genetic features of Chinese patients with HD. A) Frequency distribution of age at onset (AAO) in Chinese HD patients $(n=242)$. The AAO occurred mainly on 40 years of age. B) Spectrum of manifest HD with initial symptom $(n=242)$. Chorea was the most frequent initial symptom in manifest $\mathrm{HD}$, accounting for $80.6 \%$. The others were atypical motor symptom and cognitive or psychiatric symptom. C) Frequency distribution of CAG repeats number with normal and expanded range in affected individuals with HD $(n=306)$. D) Relationship between AAO and number of CAG repeats on the larger allele of $H T T$ gene $(n=242)$. The CAG repeats could explain approximately $74 \%$ of variance in AAO.

\section{Atypical HD patients}

\section{$\underline{\text { Juvenile HD(JHD) }}$}

Fifteen had juvenile onset (mean $14.5 \pm 4.8$ years, range 4- 20 years) with a mean CAG value of 67.0 repeats (Table S2). Four cases had been reported previously [10]. The initial symptoms of these patients were variable, including gait instability $(n=4)$, chorea $(n=4)$, intellectual decline $(n=2)$, tics $(n=1)$, tremor $(n=1)$, depression $(n=1)$ and dystonia $(n=1)$, paroxysmal seizure $(n=1)$. Three cases had no family history. Inheritance was paternal in 9 cases $(75 \%)$ and maternal in $3(25.0 \%)$. Diagnostic delay in JHD patients was 6.4 \pm 6.9 (range 1-27) years (Table 2).

\section{$\underline{\text { Elder } H D}$}

Seven patients developed symptoms at the age of 60 years or older (mean 63.7 \pm 4.8 years, range $60-71$ years) (Table S3). Six had chorea as their initial symptoms and the other one had depression five years prior to the onset of chorea. Nearly all elder HD patients complained of memory decline, some also had dysarthria and irritability. Four $(66.7 \%)$ had paternal and $2(33.3 \%)$ maternal inheritance, whereas one had unknown family history. Expanded CAG triplet number was relatively small (mean $39.3 \pm 0.9$, range 38-41) (Table 2). Diagnostic delay was $5.8 \pm 3.2$ years.

\section{$\underline{\text { Homozygous HD }}$}

Three homozygotes for elongated CAG repeats were found in our cohort (Table S4). One was reported in 2012 as first homozygous HD (asymptomatic individual) in China with CAG repeats of 37/42 [11]. Two additional cases were subsequently found with CAG repeats of 36/47 and 37/56, respectively. The reported female (CAG= 37/42) [11] was still asymptomatic at 48 years of age (in 2018). The second patient with $37 / 56$ CAG repeats suffered from developmental delay and intellectual 
decline starting at 14 years old. Five years later, she developed chorea and gait instability. The third one (CAG=36/47) first presented with chorea at the age of 40 years. Afterwards, he developed memory decline at 44 years, and irritability, dysarthria and dysphagia 4 years later. For the three homozygotes, the mean CAG repeat length of the longer allele was $48.3 \pm 7.1$, ranging from 42 to 56 repeats. The mean AAO and diagnostic delay was $29.5 \pm 14.8$ years and $6.5 \pm 4.9$ years $(n=2)$, respectively.

Table 3. Median AAO for each number of CAG repeats in Chinese patients and Dutch patients.

\begin{tabular}{|c|c|c|c|c|c|c|}
\hline \multirow{2}{*}{ CAG Repeats } & \multicolumn{3}{|c|}{ Chinese cohorts $(n=232)$} & \multicolumn{3}{|c|}{ Dutch cohort (n=755) } \\
\hline & No. of patients & Median AAO (y) & $95 \% \mathrm{CI}$ & No. of patients & Median AAO (y) & $95 \% \mathrm{CI}$ \\
\hline$<=40$ & 13 & 60 & $59-62$ & 21 & 68 & $55-81$ \\
\hline 41 & 19 & 50 & $45-53$ & 28 & 65 & $58-72$ \\
\hline 42 & 32 & 50.5 & $47-52$ & 90 & 64 & $60-68$ \\
\hline 43 & 32 & 49.5 & $44-49$ & 80 & 60 & $56-64$ \\
\hline 44 & 27 & 42 & $40-44$ & 101 & 55 & $52-58$ \\
\hline 45 & 22 & 39.5 & $37-41$ & 104 & 50 & $47-53$ \\
\hline 46 & 17 & 38 & $33-40$ & 100 & 45 & $43-47$ \\
\hline 47 & 14 & 35.5 & $32-42$ & 63 & 41 & $39-43$ \\
\hline 48 & 13 & 33 & $31-39$ & 50 & 37 & $35-39$ \\
\hline 49 & 9 & 32 & $26-45$ & 34 & 38 & $36-40$ \\
\hline 50 & 5 & 31 & $20-39$ & 19 & 34 & $30-38$ \\
\hline 51 & 2 & 29 & $4-54$ & 8 & 33 & $27-39$ \\
\hline 52 & 5 & 28 & $22-34$ & 11 & 29 & $19-29$ \\
\hline$>=53$ & 22 & 19.5 & $16-23$ & 46 & 23 & $20-26$ \\
\hline
\end{tabular}

CI: Confidence Interval. Y: years

\section{Distribution and instability features of CAG repeats}

Data on CAG repeat numbers were available for 309 persons with HD. The three homozygotes mentioned above were excluded. The range of triplet repeats in the normal allele was $11-26$ (mean 17.6 \pm 1.6 ) while in the expanded allele, the range was $37-104$ (mean $45.6 \pm 6.9$ ) (Fig. 2C).

To analyze the CAG instability during the germline transmission, we selected 46 parent-child pairs from our cohort, including 23 father-child pairs from 23 families and 23 mother-child pairs from 20 families (as one mother had three children). The gender of the affected parent was an important factor affecting the CAG repeats instability. The mean CAG repeat number was $43.4 \pm 3.3$ in father group and $46.4 \pm 10.4$ in corresponding child group, expanding by $3.0 \pm 7.9$ repeats during father-child transmission. In mother-child transmission, the CAG expanded by $0.9 \pm 2.6$ repeats with the CAG repeat number of $44.3 \pm 2.7$ in the mother group and $45.2 \pm 3.2$ in the corresponding child group. There was a difference in the father-child transmission $(\mathrm{p}=0.014)$ but not in the mother-child transmission $(\mathrm{p}=0.145)$. By adding together, the CAG repeat length was more significantly longer in the offspring group $(45.8 \pm 7.6)$ than the parent group $(43.8 \pm 3.0)(\mathrm{p}=0.005)$.

As shown in Table S5, on HD chromosomes, intergenerational CAG changes occurred in $71.7 \%$ (33/46) of meiosis, with contraction accounting for $23.9 \%$ (11/46) and expansion for $47.8 \%$ (22/46). In the remaining $28.3 \%$ (13/46) intergenerational transmissions, the CAG number was unchanged. Among 22 expanded transmissions, only two $(9.1 \%)$ transmission expanded greater than 7 repeats and they were both paternal inheritances. The expansion of CAG repeats ranged from 1 to 37 whereas contraction ranged from -3 to -1 . Paternal transmitted mutant $H T T$ alleles showed similar proportion compared with the maternal transmitted ones. 
Table 4. The number of CAG repeats, AAO and initial symptoms among different countries.

\begin{tabular}{llllllll}
\hline Genetic and clinical features & Mainland & Thai [14] & Dutch [12] & Korean [17] & Mexican [16] & African [6] \\
\hline Expanded CAG & Mean & 46.1 & 43.5 & 46.0 & 45.4 & 47.2 & 44.5 \\
& SD & 7.5 & 3.0 & 4.0 & 4.7 & 5.4 & 4.0 \\
& Range & $38-104$ & $39-48$ & $39-71$ & $40-58$ & $37-106$ & $37-57$ \\
P value & & $/$ & 0.15 & 0.80 & 0.58 & $0.01 *$ & 0.21 \\
AAO (years) & Mean & 40.3 & 37.8 & 47.0 & 46.5 & 37.4 & $\mathrm{~N}$ \\
& SD & 11.9 & 8.3 & 15.0 & 12.7 & 12.9 & $\mathrm{~N}$ \\
Pange & $4-71$ & $27-58$ & $4-86$ & $24-69$ & $2-78$ & $\mathrm{~N}$ \\
Initial symptom & Motor & $88.8 \%$ & $\mathrm{~N}$ & $\mathrm{~N}$ & $89 \% \#$ & $53.0 \%$ & $\mathrm{~N}$ \\
& Behavior & $6.2 \%$ & $\mathrm{~N}$ & $\mathrm{~N}$ & $36 \% \#$ & $30.0 \%$ & $\mathrm{~N}$ \\
\hline
\end{tabular}

$\mathrm{N}$ : means the article didn't provide. *P value was calculated when compared our cohort data with other countries'.

\#: The article of Korea counted initial symptom by first visit

\section{Correlations between CAG, AAO and initial symptoms}

Correlations between the AAO, CAG and initial symptoms were assessed in 242 cases. To explore whether CAG repeat length and AAO differed with respect to initial symptoms, we divided patients into motor, cognitive and psychiatric groups. The CAG repeat number was larger in cognitive group $(52.8 \pm 11.6)$ than motor group $(46.0 \pm 7.4)(\mathrm{p}=0.03)$ and psychiatric group $(44.4 \pm 6.3)(\mathrm{p}=0.02)$. Accordingly, the AAO of cognitive group $(25.1 \pm 9.1$ years) was earliest compared with motor group $(40.5 \pm 11.8$ years $)(\mathrm{p}=0.0003)$ and psychiatric group $(40.2 \pm 11.1$ years $)(\mathrm{p}=0.0035)$. This suggested that patients presented with cognitive impairment first had larger CAG number and earlier AAO. Further, we found chorea become more predominant with the increasement of AAO, from $26.7 \%$ (4/15) in JHD, $83.6 \%$ (184 in 220) in adult $\mathrm{HD}$ to $85.7 \%$ (6 in 7) in elder HD.

The AAO was inversely correlated with the expanded CAG repeat number $(\mathrm{p}<0.0001)$, and the $\mathrm{CAG}$ repeat lengths accounted for up to $74 \%$ of the variance in AAO $\left(\mathrm{r}=-0.86, \mathrm{R}^{2}=0.74\right)$ using the exponential regression analysis (Fig. 2D). We then separately tested their correlation in juvenile HD, adult HD and elder HD, and the $\mathrm{R}^{2}$ was $0.63,0.49$, and 0.05 , respectively. Strong associations were detected both in juvenile HD and adult HD (both $\mathrm{p}<0.001$ ). However, no correlation was found between CAG repeats and AAO in elder HD $(p=0.31)$. This indicated that the AAO of elder HD was not affected by the CAG repeat lengths.
To predict the AAO more accurately, we divided patients into groups according to the number of CAG repeats and calculated each median AAO. We then compared our result with that of Dutch population (Table 3) [12]. Median AAO in Chinese population was earlier than that in Dutch population by each number of CAG repeats.

\section{DISCUSSION}

Previous studies have suggested that the length of CAG repeats in normal HTT chromosomes is associated with the prevalence of HD in distinct populations. In a population with higher prevalence of $\mathrm{HD}$, the size of normal CAG repeats was generally larger [13]. Our study showed that the mean CAG repeats of normal allele was $17.6 \pm 1.6$ (range 11-26), which is lower than in the European population $(18.4 \pm 3.7$, range $8-35, \mathrm{n}=479$, $\mathrm{p}<0.001)$ [14]. Of note, normal alleles and intermediate alleles in the present study are limited to those determined in clinical samples of HD, which may result in an ascertainment bias. In our previous study, 483 healthy Chinese controls were sequenced in the HTT gene region and the mean CAG repeat number was $18.9 \pm 2.57$ (range 9-35) as well as the frequency of intermediate alleles was $2.5 \%(24 / 966)$ [15].

The size of CAG triplet on expanded chromosomes was within a range of 38 to 104 (mean $46.1 \pm 7.5$ ) and the mean AAO was $40.3 \pm 11.9$ (range 4-71) years (Table 4). Mexicans [16] had longer CAG length $(47.2 \pm 5.4, \mathrm{n}=691)$ 
than in our cohort. Accordingly, they had earlier AAO $(37.4 \pm 12.9$ years $)(\mathrm{p}=0.002)$. However, Korean and Dutch population had similar CAG length $(45.4 \pm 4.7$, $\mathrm{n}=36$ [17]; $46.0 \pm 4.0, \mathrm{n}=614$ [12]) to our cohort $(\mathrm{p}=0.6$, 0.8 , respectively), but their AAO was later than in our cohort with Korean population $(46.5 \pm 12.7$ years $)$ $(\mathrm{p}=0.004)$ and Dutch population $(47.0 \pm 15.0$ years $)$ $(\mathrm{p}<0.001)$. This may be caused by genetic differences with distinct modifiers present in one ethnic population but not in the other. The comparison of CAG length and AAO is shown in Table 4.

The negative correlation between CAG repeat length and AAO has been found in many studies in different populations and confirmed here again $[12,16]$. In our cohort, $74 \%$ of the variation for AAO could be explained by the CAG repeat number, which is higher compared with a Belgian cohort (50.4\%) [18] and a Venezuelan cohort (72\%) [19]. This indicates that other genetic and environmental factors also contribute to the remaining variation of AAO. A study on the monozygotic twins also suggested that different living conditions and individual habits might influence AAO [20].

We then analyzed various onset symptoms and found that the symptoms were quite diverse in juvenile onset HD. However, in adult HD and elder HD, the onset symptoms were relatively simple, usually including chorea-like symptoms. This suggests that larger CAG repeats (generally >60) cause initial symptoms of great variety. Movement symptoms were the predominant initial symptoms among Chinese HD patients (89\%), Korean patients (89\%) [17], American (69\%) [21] and Mexican (53\%) [16]. Notably, there were only 23 (9.5\%) patients whose initial symptoms were cognitive decline or psychiatric symptoms in our study. This was much lower than previously reported, estimated at approximately $33 \%$ in patients with Mexican origin [16].

In our cohort median duration of disease was 11 years, which is shorter than in the Leiden Roster, in which median survival in $800 \mathrm{HD}$ patients was 16.2 (range 2-45) years from motor symptom onset [2]. Furthermore, disease duration was independent of $\mathrm{AAO}$, which is confirmed in our analysis. Another cohort containing 4,448 DNA samples and 848 detailed disease cases with duration data also showed that the CAG repeats number determined age at death rather than disease duration [22]. Unexpectedly, our median disease duration was far less than reported cases in Europe and North America. On the one hand, it may be caused by the relatively low standard of living condition and lack of access to health-care services in the past. On the other hand, this difference may be related to uncertainties in AAO ascertainment.

Frequency of juvenile onset HD cases (AAO $<21$ years) and homozygous HD in other studies are 4\%$10 \%$ [23-25] and 0.1\%-0.4\% [26], respectively. In our study, the corresponding frequencies were $6.2 \%(15 / 242)$ and $0.9 \%(3 / 322)$, which is in a similar range. The lack of family history in a subgroup of our cohort, designated to uncertain family history, may be due to earlier death of the parents [27].

In summary, this study presents the clinical characteristics and genetic profile of HD in the Han Chinese population. This constitutes a solid foundation for future clinical, genetic and therapeutic investigation of HD in China. However, there are some limitations of this study. First, the participants were recruited from three centers located at the Southeastern China, thus there might be some population bias. Second, because of the low prevalence of HD in China, many physicians or even general neurologists lack the knowledge for accurate diagnosis. As a result, the diagnosis of many patients is delayed, and the prevalence of HD and the duration of disease is likely to be underestimated. Further studies with additional patients are needed for a more comprehensive and accurate epidemiological survey of HD in Mainland China.

\section{Conflict of interest statement}

Authors report no potential conflicts of interest.

\section{Acknowledgements}

We would like to thank all participants for their supports and willingness to participate in this study. Work in the three centers has been done in the context of the Chinese Huntington's Disease Network. This study was supported by a grant from the National Natural Science Foundation of China to Zhi-Ying Wu (81125009), Key Research and Development Project of Zhejiang Province (2019C03039), and the research foundation for distinguished scholar of Zhejiang University to Zhi-Ying Wu (188020-1938 10101/089).

\section{Supplementary Materials}

The Supplemenantry data can be found online at: www.aginganddisease.org/EN/10.14336/AD.2018.0911

\section{References}

[1] Li HL, Zhang YB, Wu ZY (2017). Development of Research on Huntington Disease in China. Neurosci Bull, 33:312-316.

[2] Roos RA, Hermans J, Vegter-van der Vlis M, van Ommen GJ, Bruyn GW (1993). Duration of illness in Huntington's disease is not related to age at onset. J Neurol Neurosurg Psychiatry, 56:98-100.

[3] Kremer B, Almqvist E, Theilmann J, Spence N, Telenius H, Goldberg YP, et al. (1995). Sex-dependent 
mechanisms for expansions and contractions of the CAG repeat on affected Huntington disease chromosomes. Am J Hum Genet, 57:343-350.

[4] Pringsheim T, Wiltshire K, Day L, Dykeman J, Steeves T, Jette N (2012). The incidence and prevalence of Huntington's disease: a systematic review and metaanalysis. Mov Disord, 27:1083-1091.

[5] Nakashima K, Watanabe Y, Kusumi M, Nanba E, Maeoka Y, Nakagawa $\mathrm{M}$, et al. (1996). Epidemiological and genetic studies of Huntington's disease in the San-in area of Japan. Neuroepidemiology, 15:126-131.

[6] Baine FK, Kay C, Ketelaar ME, Collins JA, Semaka A, Doty CN, et al. (2013). Huntington disease in the South African population occurs on diverse and ethnically distinct genetic haplotypes. Eur J Hum Genet, 21:1120-1127.

[7] Chang CM, Yu YL, Fong KY, Wong MT, Chan YW, $\mathrm{Ng} \mathrm{TH}$, et al. (1994). Huntington's disease in Hong Kong Chinese: epidemiology and clinical picture. Clin Exp Neurol, 31:43-51.

[8] Chen YY, Lai CH (2010). Nationwide populationbased epidemiologic study of Huntington's Disease in Taiwan. Neuroepidemiology, 35:250-254.

[9] Dong Y, Sun YM, Liu ZJ, Ni W, Shi SS, Wu ZY (2013). Chinese patients with Huntington's disease initially presenting with spinocerebellar ataxia. Clin Genet, 83:380-383.

[10] Liu ZJ, Sun YM, Ni W, Dong Y, Shi SS, Wu ZY (2014). Clinical features of Chinese patients with Huntington's disease carrying CAG repeats beyond 60 within HTT gene. Clin Genet, 85:189-193.

[11] Shi SS, Lin Y, Zhao GX, Gan SR, Wu ZY (2012). A Chinese pedigree with an individual homozygous for CAG repeats of Huntington's disease. Psychiatr Genet, 22:53-54.

[12] Maat-Kievit A, Losekoot M, Zwinderman K, Vegtervan der Vlis M, Belfroid R, Lopez F, et al. (2002). Predictability of age at onset in Huntington disease in the Dutch population. Medicine (Baltimore), 81:251259.

[13] Squitieri F, Andrew SE, Goldberg YP, Kremer B, Spence N, Zeisler J, et al. (1994). DNA haplotype analysis of Huntington disease reveals clues to the origins and mechanisms of CAG expansion and reasons for geographic variations of prevalence. Hum Mol Genet, 3:2103-2114.

[14] Pulkes T, Papsing C, Wattanapokayakit S, Mahasirimongkol S (2014). CAG-Expansion Haplotype Analysis in a Population with a Low Prevalence of Huntington's Disease. J Clin Neurol, 10:32-36.

[15] Jiang H, Sun YM, Hao Y, Yan YP, Chen K, Xin SH, et al. (2014). Huntingtin gene CAG repeat numbers in Chinese patients with Huntington's disease and controls. Eur J Neurol, 21:637-642.

[16] Alonso ME, Ochoa A, Boll MC, Sosa AL, Yescas P,
Lopez M, et al. (2009). Clinical and genetic characteristics of Mexican Huntington's disease patients. Mov Disord, 24:2012-2015.

[17] Shin CW, Choi YJ, Kim M, Jeon BS (2013). Preliminary analysis of Huntington's Disease in South Korea. J Huntingtons Dis, 2:83-87.

[18] Claes S, Van Zand K, Legius E, Dom R, Malfroid M, Baro F, et al. (1995). Correlations between triplet repeat expansion and clinical features in Huntington's disease. Arch Neurol, 52:749-753.

[19] Wexler NS, Lorimer J, Porter J, Gomez F, Moskowitz C, Shackell E, et al. (2004). Venezuelan kindreds reveal that genetic and environmental factors modulate Huntington's disease age of onset. Proc Natl Acad Sci U S A, 101:3498-3503.

[20] Georgiou N, Bradshaw JL, Chiu E, Tudor A, O'Gorman L, Phillips JG (1999). Differential clinical and motor control function in a pair of monozygotic twins with Huntington's disease. Mov Disord, 14:320325.

[21] Di Maio L, Squitieri F, Napolitano G, Campanella G, Trofatter JA, Conneally PM (1993). Onset symptoms in 510 patients with Huntington's disease. J Med Genet, 30:289-292

[22] Keum JW, Shin A, Gillis T, Mysore JS, Abu Elneel K, Lucente D, et al. (2016). The HTT CAG-Expansion Mutation Determines Age at Death but Not Disease Duration in Huntington Disease. Am J Hum Genet, 98:287-298.

[23] Geevasinga N, Richards FH, Jones KJ, Ryan MM (2006). Juvenile Huntington disease. J Paediatr Child Health, 42:552-554.

[24] Cannella M, Gellera C, Maglione V, Giallonardo P, Cislaghi G, Muglia M, et al. (2004). The gender effect in juvenile Huntington disease patients of Italian origin. Am J Med Genet B Neuropsychiatr Genet, 125B:92-98.

[25] Squitieri F, Berardelli A, Nargi E, Castellotti B, Mariotti C, Cannella M, et al. (2000). Atypical movement disorders in the early stages of Huntington's disease: clinical and genetic analysis. Clin Genet, 58:50-56.

[26] Alonso ME, Yescas P, Rasmussen A, Ochoa A, Macias R, Ruiz I, et al. (2002). Homozygosity in Huntington's disease: new ethical dilemma caused by molecular diagnosis. Clin Genet, 61:437-442.

[27] Hendricks AE, Latourelle JC, Lunetta KL, Cupples LA, Wheeler V, MacDonald ME, et al. (2009). Estimating the probability of de novo HD cases from transmissions of expanded penetrant CAG alleles in the Huntington disease gene from male carriers of high normal alleles (27-35 CAG). Am J Med Genet A, 149A:1375-1381. 